\title{
Visual brain plasticity induced by central and peripheral visual field loss
}

\author{
Nicolae Sanda ${ }^{1,2,3,4,5} \cdot$ Leonardo Cerliani ${ }^{6,7,8,9} \cdot$ Colas N. Authié ${ }^{1,2,3,4} \cdot$ Norman Sabbah $^{1,2,3,4}$. \\ José-Alain Sahel ${ }^{1,2,3,4,11,12,13}$. Christophe Habas ${ }^{1,2,3,10}$. Avinoam B. Safran ${ }^{1,2,3,4,5} \cdot$ Michel Thiebaut de Schotten $^{6,7,14}$
}

Received: 13 October 2017 / Accepted: 15 June 2018 / Published online: 23 June 2018

(C) The Author(s) 2018

\begin{abstract}
Disorders that specifically affect central and peripheral vision constitute invaluable models to study how the human brain adapts to visual deafferentation. We explored cortical changes after the loss of central or peripheral vision. Cortical thickness (CoTks) and resting-state cortical entropy ( $r s-\mathrm{CoEn})$, as a surrogate for neural and synaptic complexity, were extracted in 12 Stargardt macular dystrophy, 12 retinitis pigmentosa (tunnel vision stage), and 14 normally sighted subjects. When compared to controls, both groups with visual loss exhibited decreased CoTks in dorsal area V3d. Peripheral visual field loss also showed a specific CoTks decrease in early visual cortex and ventral area V4, while central visual field loss in dorsal area V3A. Only central visual field loss exhibited increased CoEn in LO-2 area and FG1. Current results revealed biomarkers of brain plasticity within the dorsal and the ventral visual streams following central and peripheral visual field defects.
\end{abstract}

Keywords Visual plasticity $\cdot$ Cortical thickness $\cdot$ Resting-state cortical entropy $\cdot$ Central visual field loss $\cdot$ Peripheral visual field loss $\cdot$ Retinitis pigmentosa $\cdot$ Stargardt macular degeneration $\cdot$ Cytoarchitectonic areas

\section{Introduction}

Vision represents the most elaborated sensory input in the human brain. Central vision is captured at retinal level by

Electronic supplementary material The online version of this article (https://doi.org/10.1007/s00429-018-1700-7) contains supplementary material, which is available to authorized users.

Nicolae Sanda

herrsanda@gmail.com

1 Sorbonne Universités, UPMC Université Paris 06, UMR S968, Institut de la Vision, 75012 Paris, France

2 INSERM, U968, Institut de la Vision, 75012 Paris, France

3 CNRS, UMR 7210, Institut de la Vision, 75012 Paris, France

4 Centre d'investigation clinique, Centre Hospitalier National d'Ophtalmologie des Quinze-Vingts, INSERM-DHOS CIC 1423, 75012 Paris, France

5 Department of Clinical Neurosciences, Geneva University Hospital and Geneva University School of Medicine, Gabrielle-Perret-Gentil 4, 1205 Geneva, Switzerland

6 Frontlab, UPMC Univ Paris 06, Inserm, CNRS, Institut du cerveau et la moelle (ICM), Hôpital Pitié-Salpêtrière, Boulevard de l'hôpital, 75013 Paris, France

7 Brain Connectivity and Behaviour Group, Sorbonne University, Paris, France the macula, which samples about $20^{\circ}$ of the central visual field and provides a high spatial resolution. The peripheral

8 Department of Psychiatry, Academic Medical Centre, Amsterdam, The Netherlands

9 Amsterdam Brain and Cognition, University of Amsterdam, Amsterdam, The Netherlands

10 Centre de Neuroimagerie, Centre Hospitalier National d'Ophtalmologie des Quinze-Vingts, 75012 Paris, France

11 Institute of Ophthalmology, University College of London, London, UK

12 Fondation Ophtalmologique Adolphe de Rothschild, Paris, France

13 Department of Ophthalmology, School of Medicine, University of Pittsburg, Pittsburg, USA

14 Groupe d'Imagerie Neurofonctionnelle, Institut des Maladies Neurodégénératives, UMR 5293, CNRS, CEA University of Bordeaux, Bordeaux, France 
visual field is collected by the remaining of the retina and has a low spatial resolution. The distinction between central and peripheral vision is also maintained within the brain. Particularly, dorsal visual areas receive relatively more projections from areas processing peripheral visual field representations, whereas ventral visual areas are more densely connected to those processing central representations (Ungerleider and Desimone 1986; Gattass et al. 2005). Hence, the loss of central or peripheral visual field should impair in different ways the visual brain and its neuroanatomy. Yet, little is known about the anatomical consequences and compensation mechanisms occurring after central or peripheral visual deprivation.

Central visual field loss prevents the central fixation, compelling patients to employ strategies of fixation in the peripheral retina, near the limit of the field defect (Duret et al. 1999). While the rest of visual field allows for an appropriate spatial orientation and navigation, its low spatial resolution impairs drastically object, face recognition and reading (Safran et al. 1999; Boucart et al. 2010). Reversely, peripheral visual field loss excludes the use of covert visual attention, constraining the affected individuals to increase their saccade rate to laboriously explore their environment (Authié et al. 2017). Affected individuals preserve functions related to the high spatial resolution of the residual central vision such as face and small objects recognition but exhibit impaired spatial orientation (Wittich et al. 2011) and scene perception (Fortenbaugh et al. 2007), altered postural control (Berencsi et al. 2005) and increased risk of object collision during locomotion (Turano et al. 1999, 2002) due to the limited coverage of the residual visual field. However, shreds of evidences support a brain reorganisation consequent to the adjustment of these behaviours.

Preliminary findings suggest that following a visual defect, the deafferented primary visual cortex alters its connections and the residual afferented primary visual cortex reinforces preexistent functional connections (Sabbah et al. 2017). The latter is presumably an attempt to compensate for the loss of the former to sustain higher order visual mechanisms. As central or peripheral visual loss generates sensory deprivation in a part of the visual cortex, structural alterations are also expected. Indeed, previous reports noted grey matter thinning in the posterior part of the primary visual cortex induced by central visual field loss (Boucard et al. 2009; Plank et al. 2011; Hernowo et al. 2014; Prins et al. 2016), whereas peripheral visual field loss induced thinning of the anterior part of the primary visual cortex (Boucard et al. 2009; Yu et al. 2013). However, above-mentioned studies evaluated disorders such as glaucoma and age-related macular degeneration that are not limited to the eye but imply widespread neurodegenerative cerebral alterations (McKinnon 2003; Pham et al. 2006; Woo et al. 2012; Chen et al. 2013; Cheung and Wong 2014). Such approaches may have hampered the identification of differences strictly related to early visual deafferentation and further research on disorders restricted to the retina may clarify the brain modification occurring after a pure early visual deprivation.

In the current study, we investigated long-term brain changes associated with two well-described pure and progressive retinal disorders that induce bilateral, converse visual field defects-Stargardt macular degeneration for central visual loss and non-syndromic retinitis pigmentosa for peripheral visual field loss. Stargardt macular degeneration is a hereditary cone-rod dystrophy that, in advanced stages, destroys the macular region, constraining affected individuals to rely only on the peripheral vision (Stargardt 1909). Reversely, retinitis pigmentosa—a rod-cone dystrophy - primarily affects peripheral retina and results in a progressive constriction of the visual field. It leads to a "tunnel vision" stage, with retained central vision, and later, in the most advanced stage, to blindness (Donders 1857; Sahel et al. 2015).

To evaluate the effects of the remote loss of central or peripheral vision, we estimated the cortical morphology derived from measures of cortical thickness (Das et al. 2009). However, the specific cellular mechanisms underlying the variations in cortical thickness remain obscure. They may be related to neuronal apoptosis, variations in cortical myelination, alterations of the synaptic complexity or a summation of these events (Wagstyl et al. 2015; Zilles and Amunts 2015; Burge et al. 2016). To explain the eventual differences in cortical thickness, we measured the functional MRI entropy during resting state session ( $r s-\mathrm{CoEn}$ ). $r s-\mathrm{CoEn}$ is a method derived from information theory and linked to neural and synaptic complexity (Tononi 1998). According to this approach, increased entropy corresponds to higher connective properties (Sokunbi et al. 2011; Yao et al. 2013; Thiebaut de Schotten et al. 2016), while the reduction in entropy may imply synaptic and dendritic degeneration (Sokunbi et al. 2013). To intimately respect its anatomy, the occipital lobe was partitioned in its corresponding cytoarchitectonic regions using cytoarchitectonic probability maps (Amunts et al. 2000; Malikovic et al. 2007; Rottschy et al. 2007; Mohlberg et al. 2012; Caspers et al. 2013; Kujovic et al. 2013; Lorenz et al. 2015; Rosenke et al. 2017). CoTks and $r s$-CoEn extracted from each of these regions were used to perform group comparisons.

\section{Methods and materials}

\section{Participants}

The Ethics Committee (Comité de protection des personnes, Ile de France V, and Agence Nationale de Sécurité du 
Médicament et des Produits de Santé) approved the study protocol (number 12,873). Thirty-eight subjects gave their written informed consent prior to inclusion. Twelve subjects suffered from Stargardt macular dystrophy (SMD) (six women, six men; all right-handed, age range from 18 to 58 year-old, mean $38.4 \pm 12$, median 39 ). This group presented a central scotoma, $10^{\circ}-20^{\circ}$ in diameter (as evaluated by Goldmann III/4 kinetic perimetry), without foveal sparing, with a best-corrected visual acuity equal or superior to 20/400 (measured by EDTRS charts). Twelve subjects suffered from retinitis pigmentosa, tunnel vision stage (RPTV) (six women, six men; nine right-handed, age range from 18 to 62 year-old, mean $41.7 \pm 16.7$, median 40 ), and presented a central residual visual field limited to a $10^{\circ}-20^{\circ}$ diameter (as evaluated by Goldmann III/4 kinetic perimetry) with a best-corrected visual acuity equal or superior to 20/40 (measured by EDTRS charts). Additionally, fourteen normally sighted controls (seven women, seven men; all right-handed, age range from 18 to 59 year-old, $41.6 \pm 14.6$, median 41), with normal routine ophthalmological examinations were also recruited for this study. Groups were matched for age and no significant difference between groups was observed (Kruskal-Wallis, c2(2) $=0.445, p=0.801$, mean rank age 19.83 in SMD group, 20.79 in RPTV group and 19.82 in normally sighted group) (see for details Supplementary Table 1).

\section{Neuroimaging}

MRI was performed with a whole-body 3T clinical imager (Sigma Horizon) using an 8-channel head coil.

T1-weighted gradient-echo images were acquired with the following parameters TE/TR/flip angle, $3.9 / 9.5 \mathrm{~ms} / 20^{\circ}$; FOV, $25.6 \times 25.6 \mathrm{~mm}$; matrix, $512 \times 512$; source voxel size, $1.2 \times 0.5 \times 0.5 \mathrm{~mm}$; thickness, $1.2 \mathrm{~mm}$, no gap.

Additionally, 32 contiguous axial T2*-weighted gradient-echo echo-planar images (TE/TR, 93/3000 ms; FOV, $240 \times 240 \mathrm{~mm}$; matrix, $64 \times 64$; voxel size, $4 \times 3.75 \times 3.75 \mathrm{~mm}$ converted to $3 \times 3 \times 3 \mathrm{~mm}$; thickness, $4 \mathrm{~mm}$; no gap; NEX, 1) were recorded to encompass the entire brain. 184 volumes were acquired including 4 "dummy" volumes obtained at the start of the session. Scan duration was 9.25 min for the whole sequence. No explicit task was required, and subjects were instructed to keep their eyes closed.

\section{Cortical thickness analysis (CoTks)}

A registration-based method (Diffeomorphic Registration based Cortical Thickness, DiReCT) was employed to estimate the cortical thickness (Das et al. 2009) from the T1-weighted imaging dataset. The first step of this method consists in creating two-voxel thick sheets, one that lies just between the grey matter and the subcortical white matter and a second lying between the grey matter and the pia matter. Then, the former is expanded to the latter using diffeomorphic deformation estimated with ANTs (Avants et al. 2007; Klein et al. 2009; Tustison and Avants 2013). The registration produces a correspondence field that allows an estimate of the distance between the outer and inner boundaries of the grey matter ribbon, and thus cortical thickness. This approach has good scan-rescan repeatability and good neurobiological validity as it can predict, with high statistical power the age and gender of the participants (Tustison et al. 2014). All these steps were carried on automatically using BCBtoolkit (http://toolkit.bcblab.com) (Foulon et al. 2018). Average cortical thickness of the occipital lobes of each subject was also measured to account for the inter-individual variability (Ferreira et al. 2017).

\section{Entropy analysis (rs-CoEn)}

First, T1-weighted gradient-echo images were skull stripped using Brain Extraction Tool (BET) as part of the FMRIB software package (FSL, http://fsl.fmrib.ox.ac.uk). T2*weighted images were subsequently registered to the anatomical (T1-weighted) image using affine deformations. Skull-stripped T1-weighted gradient-echo images were registered to the MNI152 template (http://nist.mni.mcgil 1.ca/?p=904) using affine and diffeomorphic deformations (http://stnava.github.io/ANTs) (Klein et al. 2009; Avants et al. 2011). The latter deformations were applied to the $\mathrm{T} 1$ registered T2*-weighted images. Since the resting-state $f$ MRI signal can be heavily affected by motion, even following motion correction between temporally adjacent volumes (Van Dijk et al. 2012), we estimated the signal fluctuation associated with motion and regressed it out from the $f M R I$ data prior to the calculation of entropy. To this aim, we employed a recently developed and validated procedure based on data-driven Independent Component Analysis (ICA), termed ICA-Aroma (Pruim et al. 2015). This method performs an ICA decomposition of the data and estimates which components reflect motion-related noise in the $f$ MRI signal on the basis of a robust set of spatial and temporal features. This is made possible due the distinctiveness of the motion-related components isolated by ICA on the $f$ MRI signal (Salimi-Khorshidi et al. 2014). This approach outperforms other methods such as the regression of the motion parameter estimates, while limiting in the same time the loss in degrees of freedom (Pruim et al. 2015). Compared to spike removal methods such as scrubbing (Power et al. 2012), ICA-Aroma has the advantage of preserving the temporal structure of the $f$ MRI signal. Finally, the restingstate cortical entropy ( $r s-\mathrm{CoEn}$ ) was estimated using FSL fslstats by first binning-using a fixed amount of 1000 bins - the pre-processed $f$ MRI signal within each region of 
interest (ROI), and subsequently estimating the mean Shannon entropy over the entire ROI. Like in the case of CoTks, to account for inter-individual variability, we extracted the average cortical entropy for each subject. These values were estimated on a grey matter mask from the MNI single subject template, in turn obtained from FAST segmentation, and subsequent thresholding at 0.4 the partial volume estimate map of the grey matter.

\section{Regions of interest}

To intimately respect the anatomy and cytoarchitecture of the cerebral cortex, we used probabilistic cytoarchitectonic maps of the occipital lobe (Amunts et al. 2007; Zilles and Amunts 2010; Mohlberg et al. 2012) to extract regionspecific measures of cortical thickness and entropy. These regions included hOc1 (V1), hOc2 (V2), hOc3d (V3d), hOc4d (V3A), hOc3v (VP/V3v), hOc4v (V4/V4v), hOc5 (V5/ hMT+), hOc4la (LO-2), hOc4lp (LO-1), FG1, FG2 and FG4 (http://www.fz-juelich.de/inm/inm- 1/EN/Forsc hung/_docs/SPMAnatomyToolbox/SPMAnatomyToolb ox_node.html).

\section{Statistical analysis}

We confirmed the Gaussian distribution of the data for the three groups using the Shapiro-Wilk test (Shapiro and Wilk 1965), as well as the homogeneity of variance with the Levene test (Levene 1960).

Statistical analysis was performed with SPSS 20 (SPSS, Chicago, IL, USA). Two consecutive repeated measures ANOVAs were employed to assess differences in CoTks and $r s$-CoEn between the three groups. Cytoarchitectonic areas were considered as between subject factors and hemisphere as within subject factor. Post-hoc independent-sample $t$ tests (Bonferroni corrected for multiple comparisons) were performed when statistically appropriate.

The relation between age, visual deficit duration and the cortical thickness and cortical entropy for the entire brain and for each cytoarchitectonic ROI was analysed through linear regression in SPSS 20 (SPSS, Chicago, IL, USA).

\section{Results}

\section{Cortical thickness analysis}

Whole brain average CoTks was measured for each patient and employed in group analysis, ANOVA showing no significant group effect $[F(2,35)=0.557, p=0.578]$. Further, the measured CoTks of occipital lobe cytoarchitectonic areas were normalized with the average CoTks of the entire brain.
The ratio between each cytoarchitectonic area CoTks and brain's average CoTks was further employed in the subsequent analysis (Ferreira et al. 2017). ANOVA revealed a significant group effect for the cytoarchitectonic areas hOc1 $[F(2,35)=3.882, p=0.03]$, hOc2 $[F(2,35)=4.05$, $p=0.026], \operatorname{hOc} 3 \mathrm{~d}[F(2,35)=7.09 ; p=0.003], \operatorname{hOc} 4 d[F(2$, $35)=3.633 ; p=0.037], \operatorname{hOc} 4 \mathrm{v}[F(2,35)=5.013 ; p=0.012]$. Left hemispheric regions did not differ significantly from right hemisphere regions [occipital $\mathrm{LH}, F(2,35)=2.873$, $p=0.07$; occipital RH, $F(2,35)=2.716 ; p=0.08$ ]. Post-hoc independent-sample $\mathrm{t}$ tests (Bonferroni corrected for multiple comparisons) are summarized in Table 1 and in the following paragraphs.

\section{Visual brain CoTks in visual field loss compared to normally sighted}

All the following post-hoc comparisons were Bonferroni corrected for multiple comparisons. Compared to normally sighted we found a significant reduction of CoTks in the dorsal region hOc $3 \mathrm{~d}$ for both central $(p=0.005)$ and peripheral $(p=0.015)$ visual field defects (see Table 1$)$. In central visual field loss/SMD, we also noted significant reduction in dorsal area hOc4d ( $p=0.048)$ (see Fig. 1a1, and Table 1) and in peripheral visual field loss/ RPTV in early visual cortex [hOc1 $(p=0.036), \operatorname{hOc} 2(p=0.022)]$ and the ventral region hOc $4 \mathrm{v}(p=0.017)$ (see Fig. 1a2, Table 1 and also Supplementary Fig. 1).

\section{Visual brain CoTks differences in central and peripheral visual field loss}

There was no CoTks difference between central and peripheral visual loss (see Table 1).

\section{Resting-state cortical entropy analysis}

Whole brain average CoEn was measured for each patient and employed in group analysis, ANOVA showing no significant group effect $[F(2,35)=1.378, p=0.273]$. Further, the measured $r s$-CoEn of occipital lobe cytoarchitectonic areas was normalized with the average rs-CoEn of the entire brain. The ratio between each cytoarchitectonic area $r s$-CoEn and brain's average $r s$-CoEn was further employed in the subsequent analysis. ANOVA revealed a significant group effect for the cytoarchitectonic areas hOc4la $(F(2,35)=3.856 ; p=0.031)$ and FG1 $(F(2,35)=6.566$; $p=0.004)$. There was no significant effect for the factor hemisphere [occipital LH, $F(2,35)=0.43, p=0.654$; occipital RH, $F(2,35)=0.082 ; p=0.922]$. Note that none of the areas with altered CoTks compared to normally sighted exhibited significant $r s$-CoEn alterations. Post-hoc 


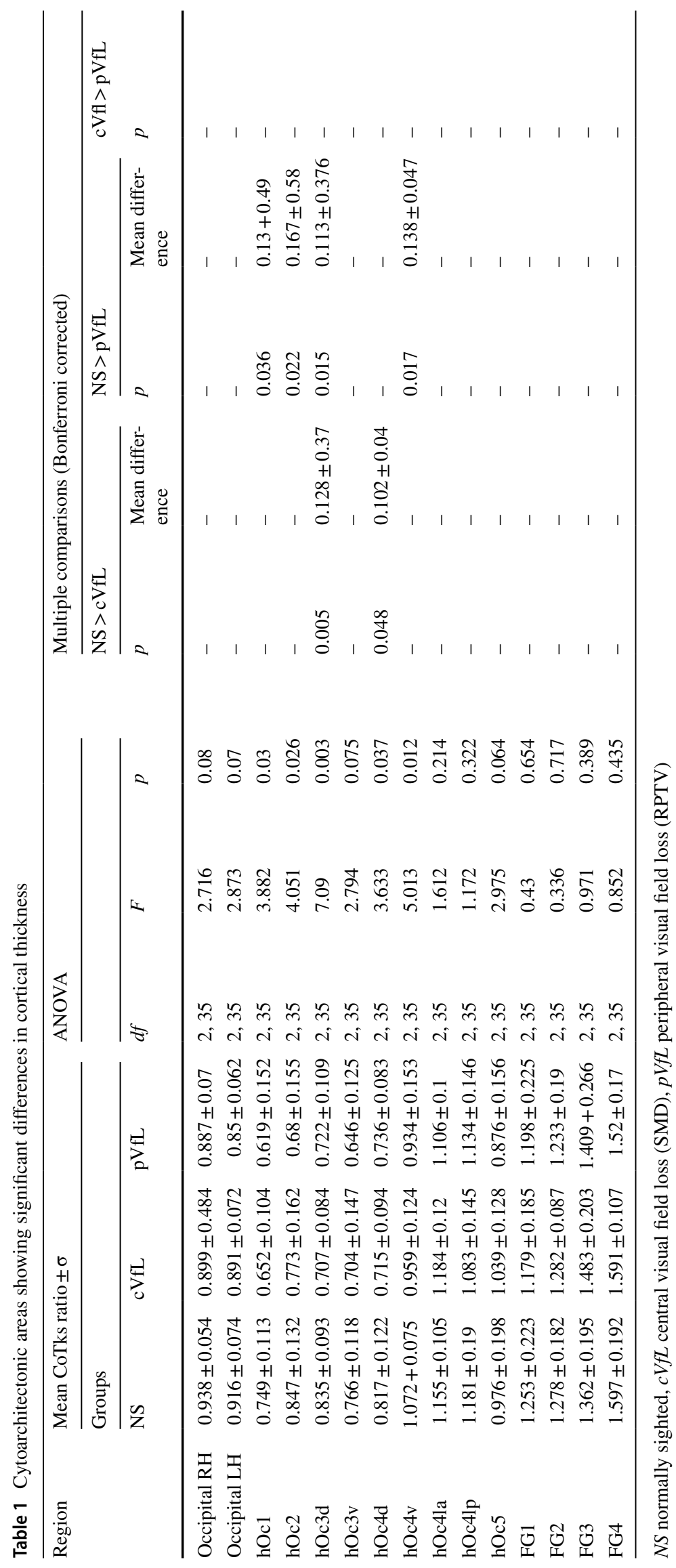




\section{A Between-groups cortical thickness analysis}

1. Cytoarchtectonic areas with reduced cortical thickness in central visual loss compared to normally sighted

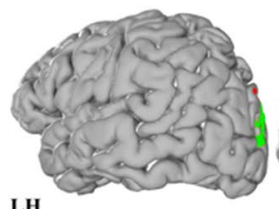

LH
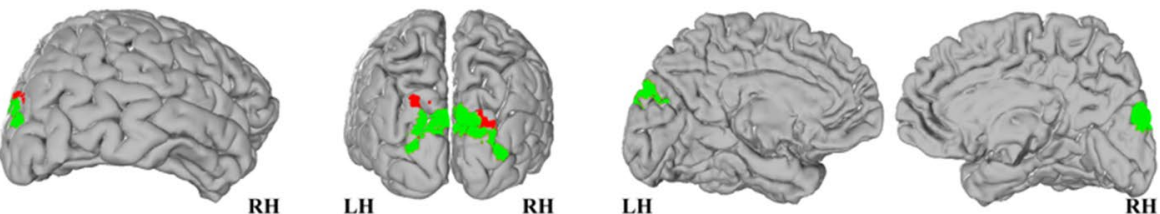

hOc3d (V3d), p= 0.005

hOc4d (V3A), p= 0.05

2. Cytoarchtectonic areas with reduced cortical thickness in peripheral visual loss compared to normally sighted

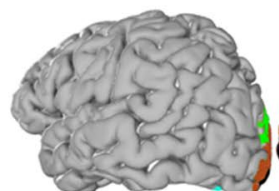

LH

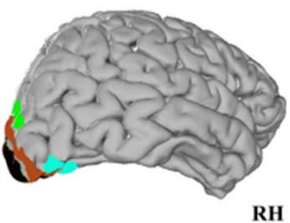

RH

hOc2 (V2), p $=0.022$

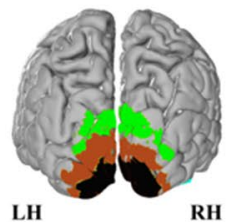

LH

RH

hOc3d (V3d), p= 0.015

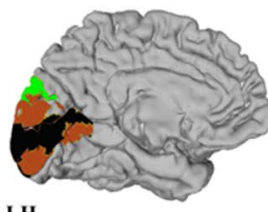

LH

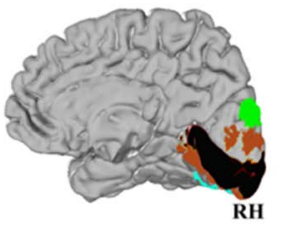

hOc4v (V4), $\mathrm{p}=0.017$

\section{B Between-groups cortical entropy analysis}

\section{Cytoarchtectonic areas with increased cortical entropy in central visual loss (SMD) compared to normally sighted}

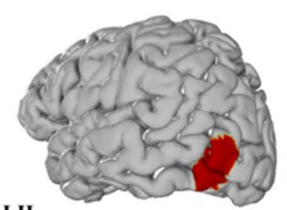

LH

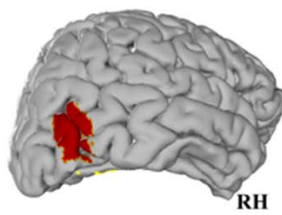

FG1 $p=0.025$

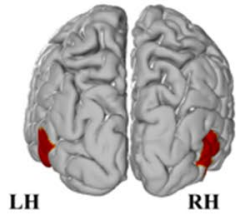

LH

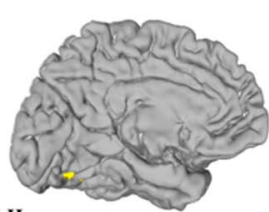

LH

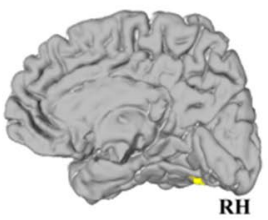

hOc4la (LO-2) p= 0.033

2. Cytoarchtectonic areas with increased cortical entropy in central visual loss compared (SMD) to peripheral visual loss (RPTV)

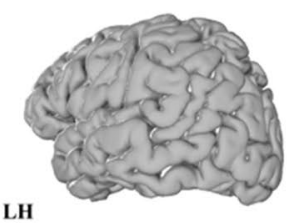

LH

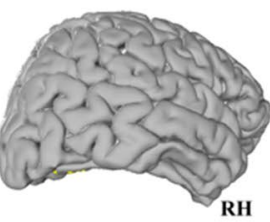

FG1 $\mathrm{p}=0.005$

Fig. 1 a Between-group analysis of cortical thickness. b Betweengroup analysis of cortical entropy. The cytoarchitectonic areas are shown on neutral brains (left hemisphere) extracted from JuBrain
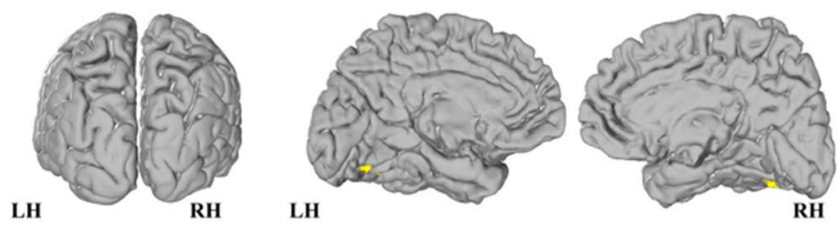

CytoViewer Atlas (https://www.jubrain.fz-juelich.de); each cytoarchitectonic group is depicted in a different colour 


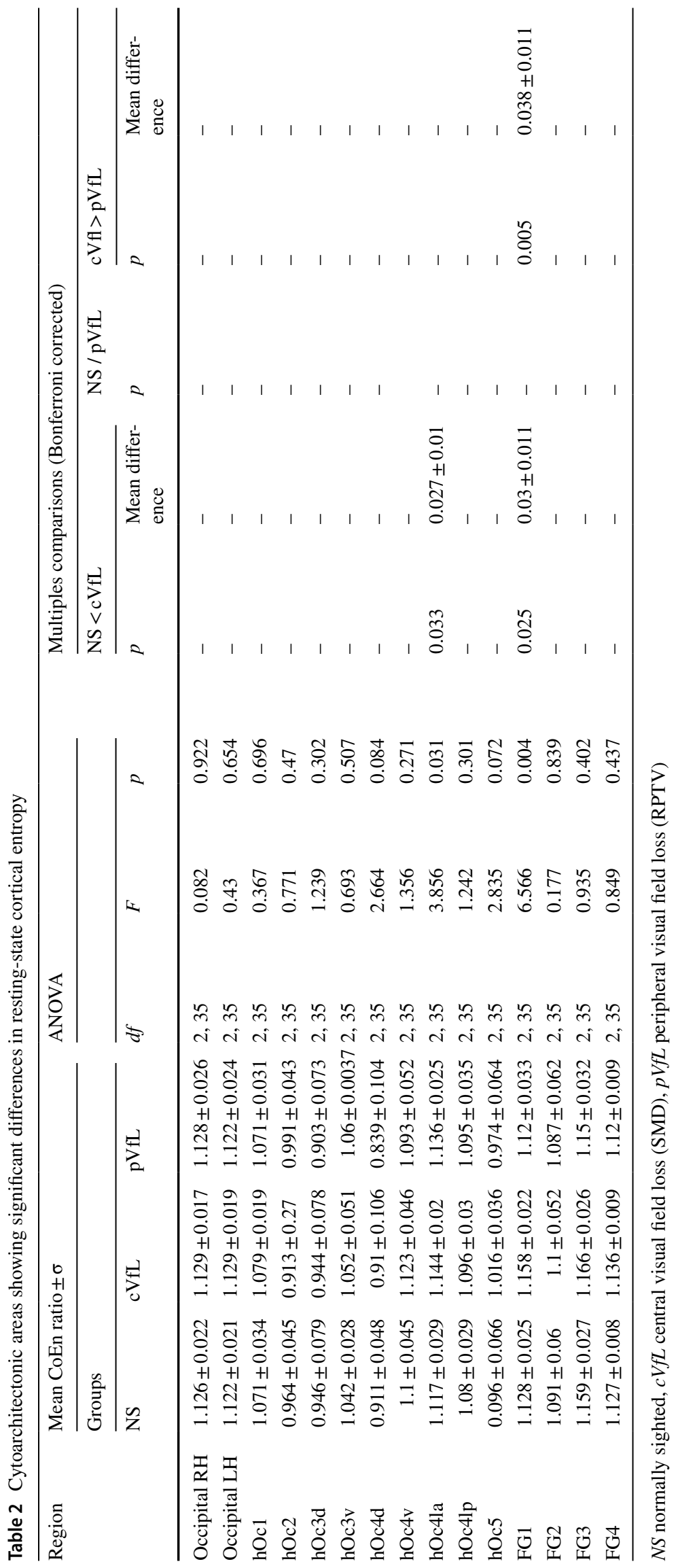


independent-sample $t$ tests (Bonferroni corrected for multiple comparisons) are summarized in Table 2 and in the following paragraphs.

\section{Visual brain rs-CoEn in visual field loss compared to normally sighted}

Compared to normally sighted we found in central visual field defect/ SMD group a significant increase of $r s$-CoEn in areas hOc4la $(p=0.031)$ and FG1 $(p=0.025)$ (see Fig. 1b.1, Table 2 and also Supplementary Fig. 2). There was no $r s$ CoEn difference between peripheral visual loss/RPTV and the normally sighted.

\section{Visual brain rs-CoEn differences in central and peripheral visual field loss}

Compared to the peripheral visual field loss/RPTV group, central visual field loss/SMD exhibited significantly higher $r s$-CoEn in the area FG1 $(p=0.005)$ (see Fig. 1b.2, Table 2 and also Supplementary Fig. 2).

\section{Impact of age and duration deficit on CoTks and rs-CoEn}

There was no impact of age and deficit duration on the CoTks and $r s-\mathrm{CoEn}$ in areas exhibiting differences between groups (for regression differences in other areas see Supplementary Tables 1 and 2). Nevertheless, we found an impact of age $(p<0.0001)$ and disease duration $(p<0.0001)$ on global CoTks, but no impact on the $r s$-CoEn.

\section{Discussion}

We assessed the impact of central and peripheral vision loss on the cortical morphology (i.e. cortical thickness, CoTks) and neural and synaptic complexity (i.e. $r s$-fMRI entropy, $r s$-CoEn). Three findings emerge from our work. First, compared to normally sighted both groups with visual field defects exhibited reduced CoTks in the dorsal region hOc3d; peripheral visual field defect group also presented reduced CoTks in early visual cortex (hOc1 and hOc2) and the ventral region hOc4V, while central visual field defect group in the dorsal region hO4d. Second, compared both to normally sighted and peripheral visual field defect groups, central visual field defect group showed increased $r s$-CoEn in FG1 area; also, compared with normally sighted, central visual field defect group exhibited increased $r s-\mathrm{CoEn}$ in hOc4la area. Finally, areas with altered CoTks had normal $r s$-CoEn and conversely.

\section{Differences in cortical thickness}

Compared to normally sighted only the subjects with peripheral visual loss showed decreased CoTks in hOc1 and hOc2, which correspond to the functional regions V1 and V2 of the early visual cortex (Amunts et al. 2000). Previous studies reported a decreased CoTks in the early visual cortex for both central and peripheral visual loss (Boucard et al. 2009; Plank et al. 2011; Yu et al. 2013; Hernowo et al. 2014; Prins et al. 2016). In our study, only peripheral visual loss was associated with a thinning of the early visual cortex. This difference might be explained by the peculiarities of the retinal degeneration in retinitis pigmentosa. Retinitis pigmentosa is a pan-retinal, rod-cone degeneration and in the tunnel stage, patients exhibit not only the loss of all receptors in the peripheral retina, but also the loss of rods in central retina coupled to a more limited degeneration of central cones (translated by a reduced visual acuity, Sahel et al. 2015). Moreover, rod loss might directly impact certain photopic vision processes such as cone-driven, horizontal cell mediated surround inhibition (Szikra et al. 2014) or mesopic (dim-light) vision processes such as rod-cone or rod-rod gap-junction coupling presumed to help identifying dark objects moving through the visual field (Tsukamoto et al. 2001; Volgyi 2004; Ribelayga et al. 2008; Bloomfield and Völgyi 2009). SMD, on the other hand, associates a photoreceptor loss that is solely localized to central retina (Meunier and Puech 2012). Hence, the loss of cortical thickness in V1 and V2 we report herein, suggests that retinal degeneration in retinitis pigmentosa has a greater trophic impact on early visual areas. The loss of the peripheral vision represents the loss of an extensive visual field area and affects the output of numerous wide-field retinal informational channels (Ölveczky et al. 2003; Roska and Werblin 2003; Hosoya et al. 2005; Münch et al. 2009; Masland 2012). Yet poorly understood these channels might play an important role in the functioning of the early visual cortex.

The reduced hOc3d CoTks in both visual field defects when compared to normally sighted, suggest a comparable contribution of central and peripheral visual field to the dorsal portion of V3 (V3d), which is canonically included in the dorsal stream (Kujovic et al. 2013). Anatomical and functional data indicate that the primarily role of V3d area is the processing kinetic information (Felleman et al. 1997; Gegenfurtner et al. 1997; Rosa and Manger 2005), the extraction of kinetic contours (Zeki 2003), and 3D form (Vanduffel 2002). Moreover, V3d has the particularity that its retinotopical map represents only the lower quadrant of the visual field, while the upper quadrant is being represented in the ventral part of $\mathrm{V} 3$, area $\mathrm{V} 3 \mathrm{v}(\mathrm{hOc} 3 \mathrm{v}$, Rottschy et al. 2007; Kujovic et al. 2013). It is possible that the spatial nature of information processing in hOc $3 \mathrm{~d} /$ V3d is responsible for the decreased CoTks observed with 
both central and peripheral visual field loss. Indeed, the build-up of an accurate referential system, essential for functions such as stereopsis (i.e. 3D perception), requires both central vision, which provides high spatial resolution and fixation, and peripheral vision, which provides widefield sampling (Goldstein and Clahane 1966; Luria 1971; Dessing et al. 2012). In central visual loss, the physiological foveal fixation lacks and compels to fixation in the vicinity of the visual field defect, in the residual functional periphery. These eccentric fixation loci (usually multiple) are used both for detection (Duret et al. 1999) and visuomotor coordination (Timberlake et al. 2012) and occur in different retinal positions for each eye. These peculiarities lead to an inadequate extraction of fixation disparities (Wheatstone 1962) impairing the very mechanism of stereopsis. In peripheral visual field loss, foveal vision, physiologic fixation and visual acuity are preserved, but stereopsis is nevertheless impaired through mechanisms such as a non-uniform drifting of the two eyes in the absence of the peripheral visual field superposition, the loss of fusion due to brief occlusions (i.e. eye-blinks, Fender and Julesz 1967) or "empty-field myopia" (i.e. accommodation impairment due to increased amplitude oscillation of accommodation in the absence of peripheral clues resulting in increased difficulty for detection, Whiteside 1957; Campbell et al. 1959). Hence, cortical thickness reduction of hOc3d CoTks in both visual field defects may account for the important contribution of central and peripheral visual fields to the functioning of this brain area.

Interestingly, compared to normally sighted, central visual field loss also exhibited decreased CoTks in the dorsal area hOc4d, corresponding to the functional region $\mathrm{V} 3 \mathrm{~A}$. This area seems to be involved in the processing of kinetic and static 3D shapes (Georgieva et al. 2009), especially contour curvature (Caplovitz and Tse 2006), stereoscopic and chromatic motion (McKeefry et al. 2010; Anzai et al. 2011), perceptual stability during eye movements (Braddick et al. 2001; Fischer et al. 2012), the prediction of the visual motion (Maus et al. 2010), its structural damage commonly resulting in simultanagnosia, namely the inability to interpret complex visual displays despite the preserved capacity to recognize single objects (Coslett and Saffran 1991). Impaired fixation and stereoscopic vision in patients with central visual loss may account for the CoTks loss in this area.

Another intriguing result was the decreased CoTks in area hOc4v in peripheral field loss, when compared to normally sighted. Area hOc4, to the best of our knowledge, probably corresponds to human V4 (hV4) or at least to its ventral subdivision $\mathrm{V} 4 \mathrm{v}(\mathrm{hV} 4 \mathrm{v})$. The role of $\mathrm{hV} 4 \mathrm{in}$ colour perception is still debated (Bartolomeo et al. 2014), but its central participation in the figure-ground segmentation through the integration of multiple stimulus properties (i.e. contour, shape, texture, motion, colour, disparity) by bottom-up salience driven attentional mechanism or topdown proactive spatial or feature selection makes consensus (Reynolds and Desimone 2003; Qiu et al. 2007; Poort et al. 2012; Roe et al. 2012). The severely constricted visual field, resulting from the loss of the peripheral visual field, may limit covert visual attention and the sensory input in area hOc4 and consequently impact its CoTks.

\section{Differences in cortical entropy}

Compared to normally sighted, central visual field loss group exhibited increased $r s$-CoEn in area hOc4la that likely corresponds to functionally defined LO-2 region (Larsson and Heeger 2006) involved in shape processing, object and face recognition, visual attention, action observation, visual tracking, spatial location discrimination, mental imagery and subjective emotional picture discrimination (Malikovic et al. 2016). The increased $r s$ CoEn in area hOc4la/ LO-2 suggests an adaptive increase in synaptic complexity points in this area, which is crucial for shape perception, figure-ground segregation and visuomotor coordination (Malikovic et al. 2016). Moreover, in a previous study exploring the resting state functional connectivity of central and peripheral V1 in the exact populations explored here, we found that in central visual field loss, afferented peripheral early visual cortex exhibited increased functional connectivity with LOC compared to the corresponding region in normally sighted (Sabbah et al. 2017). Therefore, in central visual loss, the increased $r s$-CoEn in LO-2 might be linked to the increased functional connectivity of this area with the residually afferented peripheral early visual cortex.

Subjects with central visual field loss presented increased $r s$-CoEn in area FG1 when compared to normally sighted and peripheral visual field loss participants. This area, located in the posterior part of the fusiform gyrus, medial to the middle fusiform sulcus (Caspers et al. 2013; Lorenz et al. 2015) exhibits a bias for the peripheral visual field representations. More precisely, FG1 and the anteriorly situated FG3 overlap with places, inanimate large objects and peripheral biased representations (Lorenz et al. 2015). This line of evidences suggests that the observed difference in $r s$-CoEn may relate to an enhanced peripheral visual field treatment in FG1 area to compensate for the central visual field loss. In accordance with this compensation hypothesis, peripheral early visual cortex in central visual field loss showed increased resting-state functional connectivity with fusiform gyrus compared to peripheral early visual cortex in normally sighted. 


\section{Combined cortical thickness and cortical entropy data}

Interestingly, the regions with altered CoTks had normal $r s$-CoEn and conversely. Limitations aside, reduced CoTks with normal $r s$-CoEn may indicate a possible mixture of cell shrinkage with preserved synaptic complexity of the remaining networks. On the other hand, increased $r s$-CoEn might correspond to a brain plasticity effect induced by an increased visual afference of the spared visual field.

\section{Limitations}

Cytoarchitectonic areas are highly variable across subjects (Amunts et al. 1999). Herein, we extracted occipital cytoarchitectonic areas from an atlas based on observerindependent probabilistic mapping of ten post-mortem brains (Mohlberg et al. 2012; Zilles and Amunts 2015). To reduce the effect of inter-individual variability, CoTks and $r s$-CoEn were sampled only in voxels where the same cytoarchitectonic area overlaps in more than five out of ten of the post-mortem brains investigated.

Myelin density in the cortex and various technical parameters (field strength, tissue segmentation methods, smoothing, etc.) may influence the MRI measure of cortical thickness and lead to incorrect estimations (Glasser et al. 2014). These effects can be particularly deceptive in disorders that impact myelination (i.e. multiple sclerosis) or in physiological states exhibiting different degrees of cortical myelination (i.e. development or ageing, Westlye et al. 2010; Zilles and Amunts 2015). Unfortunately, we could not assess cortical myelination with T1-weighted images. Future research investigating T1 intensity may shed light on that matter (Turner et al. 2008; Stüber et al. 2014).

Resting-state $f$ MRI signal is notoriously affected by motion (Van Dijk et al. 2012). Increased movements would virtually increase measures of entropy. To reduce this effect, we regressed out the motion-related signal from the $f \mathrm{MRI}$ data before the calculation of entropy. In this way, we maximised the likelihood that the entropy measures reflect the spontaneous hemodynamic fluctuations related to brain activity.

We noted an overall effect of age and in patient groups also of disease duration on brain CoTks, but not on the CoEn. However, we found no effect of age and disease duration on CoTks in areas exhibiting differences between groups. The lack of effect of age and disease duration on the CoTks of the studied cytoarcitectonic areas might be due to limited size of our studied groups. In addition, the absent impact of age and disease duration on CoEn might reflect the plastic changes resulting in a preserved synaptic complexity.

\section{Conclusion}

Overall, central and peripheral visual loss induced complex structural changes unpredicted by the canonical segregation central vision-ventral visual field, peripheral vision-dorsal visual field. We found that central visual field loss induces a thinning in dorsal stream areas hOc3d (V3d) and hOc4d (V3A) and peripheral visual field loss in early visual cortex (hOc1/V1 and hOc2/V2), dorsal stream area hOc3d (V3d) and ventral stream area hOc4v (V4). Central visual field loss also induces an increase in entropy in areas hOc4la (LO-2) and FG-1 reflecting possible alternative, compensatory processing. These results offer a new and interesting insight on the effect of central and peripheral visual field deafferentation and also invite to revisit the canonical concepts of "ventral" and "dorsal" stream. Moreover, these data suggest complex adaptive changes that should be considered in the development of new visually rehabilitation strategies, sensory substitution devices or visual restitution attempts.

Acknowledgements We would like to thank the team of the "Centre d'investigation clinique" of the "Centre Hospitalier National d'Ophtalmologie des Quinze-Vingts", particularly Saddek MohandSaïd MD and Céline Devisme PhD for their invaluable help in patients' recruitment and the members of "Forschungszentrum Jülich GmbH" for providing us with the cytoarchitectonic maps of the occipital lobe.

Funding The research leading to these results received funding from the programs: "Jeune Chercheuses et Jeunes Chercheurs" ANR-13JSV4-0001-01, "Investissements d'avenir" ANR-10-IAIHU-06, "LABEX" and "Humanis".

\section{Compliance with ethical standards}

Conflict of interest There is no conflict of interest to declare for any author excepting José-Alain Sahel, consultant for Pixium Vision, GenSight Biologics, Sanofi-Fovea, and Genesignal. However, this had no influence on the results or discussion reported in this paper.

Open Access This article is distributed under the terms of the Creative Commons Attribution 4.0 International License (http://creativeco mmons.org/licenses/by/4.0/), which permits unrestricted use, distribution, and reproduction in any medium, provided you give appropriate credit to the original author(s) and the source, provide a link to the Creative Commons license, and indicate if changes were made.

\section{References}

Amunts K, Schleicher A, Bürgel U et al (1999) Broca's region revisited: cytoarchitecture and intersubject variability. J Comp Neurol 412:319-341. 10.1002/(SICI)10969861(19990920)412:2<319::AID-CNE10>3.0.CO;2-7

Amunts K, Malikovic A, Mohlberg H et al (2000) Brodmann's areas 17 and 18 brought into stereotaxic space-where and how variable? Neuroimage 11:66-84. https://doi.org/10.1006/nimg.1999.0516 
Amunts K, Schleicher A, Zilles K (2007) Cytoarchitecture of the cerebral cortex-more than localization. Neuroimage 37:1061-1065. https://doi.org/10.1016/j.neuroimage.2007.02.037

Anzai A, Chowdhury SA, DeAngelis GC (2011) Coding of stereoscopic depth information in visual areas V3 and V3A. J Neurosci 31:10270-10282. https://doi.org/10.1523/JNEUR OSCI.5956-10.2011

Authié CN, Berthoz A, Sahel J-A, Safran AB (2017) Adaptive gaze strategies for locomotion with constricted visual field. Front Hum Neurosci 11:387. https://doi.org/10.3389/fnhum.2017.00387

Avants B, Duda JT, Zhang H, Gee JC (2007) Multivariate normalization with symmetric diffeomorphisms for multivariate studies. In: Medical image computing and computer-assisted intervention-MICCAI 2007. Springer, Berlin Heidelberg, pp 359-366

Avants BB, Tustison NJ, Song G et al (2011) A reproducible evaluation of ANTs similarity metric performance in brain image registration. Neuroimage 54:2033-2044. https://doi.org/10.1016/j. neuroimage.2010.09.025

Bartolomeo P, Bachoud-Lévi A-C, Thiebaut de Schotten M (2014) The anatomy of cerebral achromatopsia: a reappraisal and comparison of two case reports. Cortex 56:138-144. https://doi. org/10.1016/j.cortex.2013.01.013

Berencsi A, Ishihara M, Imanaka K (2005) The functional role of central and peripheral vision in the control of posture. Hum Mov Sci 24:689-709. https://doi.org/10.1016/j.humov .2005 .10 .014

Bloomfield SA, Völgyi B (2009) The diverse functional roles and regulation of neuronal gap junctions in the retina. Nat Rev Neurosci 10:495-506. https://doi.org/10.1038/nrn2636

Boucard CC, Hernowo AT, Maguire RP et al (2009) Changes in cortical grey matter density associated with long-standing retinal visual field defects. Brain 132:1898-1906. https://doi. org/10.1093/brain/awp119

Boucart M, Naili F, Despretz P et al (2010) Implicit and explicit object recognition at very large visual eccentricities: no improvement after loss of central vision. Vis $\operatorname{cogn}$ 18:839-858. https://doi.org/10.1080/13506280903287845

Braddick OJ, O'Brien JMD, Wattam-Bell J et al (2001) Brain areas sensitive to coherent visual motion. Perception 30:61-72. https ://doi.org/10.1068/p3048

Burge WK, Griffis JC, Nenert R et al (2016) Cortical thickness in human V1 associated with central vision loss. Sci Rep 6:23268. https://doi.org/10.1038/srep23268

Campbell FW, Robson JG, Westheimer G (1959) Fluctuations of accommodation under steady viewing conditions. J Physiol 145:579-594

Caplovitz GP, Tse PU (2006) V3A processes contour curvature as a trackable feature for the perception of rotational motion. Cereb Cortex 17:1179-1189. https://doi.org/10.1093/cercor/bhl029

Caspers J, Zilles K, Eickhoff SB et al (2013) Cytoarchitectonical analysis and probabilistic mapping of two extrastriate areas of the human posterior fusiform gyrus. Brain Struct Funct 218:511-526. https://doi.org/10.1007/s00429-012-0411-8

Chen WW, Wang N, Cai S et al (2013) Structural brain abnormalities in patients with primary open-angle glaucoma: a study with 3T MR imaging. Invest Ophthalmol Vis Sci 54:545-554. https:// doi.org/10.1167/iovs.12-9893

Cheung CMG, Wong TY (2014) Is age-related macular degeneration a manifestation of systemic disease? New prospects for early intervention and treatment. J Intern Med 276:140-153. https ://doi.org/10.1111/joim.12227

Coslett HB, Saffran E (1991) Simultanagnosia. To see but not two see. Brain 114 (Pt 4:1523-1545. https://doi.org/10.1093/brain $/ 114.4 .1523$
Das SR, Avants BB, Grossman M, Gee JC (2009) Registration based cortical thickness measurement. Neuroimage 45:867-879. https://doi.org/10.1016/j.neuroimage.2008.12.016

Dessing JC, Rey FP, Beek PJ (2012) Gaze fixation improves the stability of expert juggling. Exp brain Res 216:635-644. https ://doi.org/10.1007/s00221-011-2967-6

Donders FC (1857) Beiträge zur pathologischen Anatomie des Auges. Albr von Graefes Arch für Ophthalmol 3:139-165. https://doi.org/10.1007/BF02720685

Duret F, Issenhuth M, Safran AB (1999) Combined use of several preferred retinal loci in patients with macular disorders when reading single words. Vision Res 39:873-879. https://doi. org/10.1016/S0042-6989(98)00179-5

Felleman DJ, Burkhalter A, Van Essen DC (1997) Cortical connections of areas V3 and VP of macaque monkey extrastriate visual cortex. J Comp Neurol 379:21-47. 10.1002/(SICI)10969861(19970303)379:1<21::AID-CNE3>3.0.CO;2-K

Fender D, Julesz B (1967) Extension of Panum's fusional area in binocularly stabilized vision. J Opt Soc Am 57:819-830. https://doi. org/10.1364/JOSA.57.000819

Ferreira S, Pereira AC, Quendera B et al (2017) Primary visual cortical remapping in patients with inherited peripheral retinal degeneration. NeuroImage Clin 13:428-438. https://doi.org/10.1016/j. nicl.2016.12.013

Fischer E, Bülthoff HH, Logothetis NK, Bartels A (2012) Human areas V3A and V6 compensate for self-induced planar visual motion. Neuron 73:1228-1240. https://doi.org/10.1016/j.neuro n. 2012.01.022

Fortenbaugh FC, Hicks JC, Hao L, Turano KA (2007) Losing sight of the bigger picture: peripheral field loss compresses representations of space. Vision Res 47:2506-2520. https://doi. org/10.1016/j.visres.2007.06.012

Foulon C, Cerliani L, Kinkingnehun S et al (2018) Advanced lesion symptom mapping analyses and implementation as BCBtoolkit. Gigascience. https://doi.org/10.1101/133314

Gattass R, Nascimento-Silva S, Soares JGM et al (2005) Cortical visual areas in monkeys: location, topography, connections, columns, plasticity and cortical dynamics. Philos Trans R Soc B Biol Sci 360:709-731. https://doi.org/10.1098/rstb.2005.1629

Gegenfurtner KR, Kiper DC, Levitt JB (1997) Functional properties of neurons in macaque area V3. J Neurophysiol 77:1906-1923

Georgieva S, Peeters R, Kolster H et al (2009) The processing of threedimensional shape from disparity in the human brain. J Neurosci 29:727-742. https://doi.org/10.1523/JNEUROSCI.4753-08.2009

Glasser MF, Goyal MS, Preuss TM et al (2014) Trends and properties of human cerebral cortex: correlations with cortical myelin content. Neuroimage 93:165-175. https://doi.org/10.1016/j.neuro image.2013.03.060

Goldstein JH, Clahane AC (1966) The role of the periphery in binocular vision. Am J Ophthalmol 62:702-706. https://doi. org/10.1016/0002-9394(66)92198-2

Hernowo AT, Prins D, Baseler H et al (2014) Morphometric analyses of the visual pathways in macular degeneration. Cortex 56:99 110. https://doi.org/10.1016/j.cortex.2013.01.003

Hosoya T, Baccus SA, Meister M (2005) Dynamic predictive coding by the retina. Nature 436:71-77. https://doi.org/10.1038/natur e03689

Klein A, Andersson J, Ardekani BA et al (2009) Evaluation of 14 nonlinear deformation algorithms applied to human brain MRI registration. Neuroimage 46:786-802. https://doi.org/10.1016/j. neuroimage.2008.12.037

Kujovic M, Zilles K, Malikovic A et al (2013) Cytoarchitectonic mapping of the human dorsal extrastriate cortex. Brain Struct Funct 218:157-172. https://doi.org/10.1007/s00429-012-0390-9 
Larsson J, Heeger DJ (2006) Two retinotopic visual areas in human lateral occipital cortex. J Neurosci 26:13128-13142. https://doi. org/10.1523/JNEUROSCI.1657-06.2006

Levene H (1960) Robust tests for equality of variances. In: Olkin I, Ghurye SG, Hoeffding W et al (eds) Contributions to probability and statistics: essays in honor of harold hotelling. Stanford University Press, Stanford, pp 278-292

Lorenz S, Weiner KS, Caspers J et al (2015) Two new cytoarchitectonic areas on the human mid-fusiform gyrus. Cereb Cortex BHV 225:1-13. https://doi.org/10.1093/cercor/bhv225

Luria SM (1971) Duction, stereoacuity and field of view.pdf. Optom Vis Sci 48:728-735

Malikovic A, Amunts K, Schleicher A et al (2007) Cytoarchitectonic analysis of the human extrastriate cortex in the region of V5/ MT+: a probabilistic, stereotaxic map of area hOc5. Cereb Cortex 17:562-574. https://doi.org/10.1093/cercor/bhj181

Malikovic A, Amunts K, Schleicher A et al (2016) Cytoarchitecture of the human lateral occipital cortex: mapping of two extrastriate areas hOc4la and hOc4lp. Brain Struct Funct 221:18771897. https://doi.org/10.1007/s00429-015-1009-8

Masland RH (2012) Another blue neuron in the retina. Nat Neurosci 15:930-931. https://doi.org/10.1038/nn.3146

Maus GW, Weigelt S, Nijhawan R, Muckli L (2010) Does area V3A predict positions of moving. Objects? Front Psychol 1:1-11. https://doi.org/10.3389/fpsyg.2010.00186

McKeefry DJ, Burton MP, Morland AB (2010) The contribution of human cortical area V3A to the perception of chromatic motion: a transcranial magnetic stimulation study. Eur J Neurosci 31:575-584. https://doi.org/10.111 $1 /$ j.1460-9568.2010.07095.x

McKinnon SJ (2003) Glaucoma: ocular Alzheimer's disease? Front Biosci 8:s1140-s1156

Meunier I, Puech B (2012) Maladie de Stargardt (Stargardt disease). In: Cohen SY, Gaudric A (eds) Rétine. Lavoisier, Paris, pp 26-48

Mohlberg H, Eickhoff SB, Schleicher A et al (2012) A new processing pipeline and release of cytoarchitectonic probabilistic mapsJuBrain. In: OHBM. Peking

Münch T, da Silveira RA, Siegert S et al (2009) Approach sensitivity in the retina processed by a multifunctional neural circuit. Nat Neurosci 12:1308-1316. https://doi.org/10.1038/nn.2389

Ölveczky BP, Baccus SA, Meister M (2003) Segregation of object and background motion in the retina. Nature 423:401-408. https:// doi.org/10.1038/nature01652

Pham TQ, Kifley A, Mitchell P, Wang JJ (2006) Relation of age-related macular degeneration and cognitive impairment in an older population. Gerontology 52:353-358. https://doi.org/10.1159/00009 4984

Plank T, Frolo J, Brandl-Rühle S et al (2011) Gray matter alterations in visual cortex of patients with loss of central vision due to hereditary retinal dystrophies. Neuroimage 56:1556-1565. https ://doi.org/10.1016/j.neuroimage.2011.02.055

Poort J, Raudies F, Wannig A et al (2012) The role of attention in figure-ground segregation in areas $\mathrm{V} 1$ and $\mathrm{V} 4$ of the visual cortex. Neuron 75:143-156. https://doi.org/10.1016/j.neuro n.2012.04.032

Power JD, Barnes KA, Snyder AZ et al (2012) Spurious but systematic correlations in functional connectivity MRI networks arise from subject motion. Neuroimage 59:2142-2154. https://doi. org/10.1016/j.neuroimage.2011.10.018

Prins D, Plank T, Baseler HA et al (2016) Surface-based analyses of anatomical properties of the visual cortex in macular degeneration. PLoS One 11:e0146684. https://doi.org/10.1371/journ al.pone. 0146684

Pruim RHR, Mennes M, Buitelaar JK, Beckmann CF (2015) Evaluation of ICA-AROMA and alternative strategies for motion artifact removal in resting state fMRI. Neuroimage 112:278-287. https://doi.org/10.1016/j.neuroimage.2015.02.063

Qiu FT, Sugihara T, von der Heydt R (2007) Figure-ground mechanisms provide structure for selective attention. Nat Neurosci 10:1492-1499. https://doi.org/10.1038/nn1989

Reynolds JH, Desimone R (2003) Interacting roles of attention and visual salience in V4. Neuron 37:853-863. https://doi.org/10.1016/ S0896-6273(03)00097-7

Ribelayga C, Cao Y, Mangel SC (2008) The circadian clock in the retina controls rod-cone coupling. Neuron 59:790-801. https:// doi.org/10.1016/j.neuron.2008.07.017

Roe AW, Chelazzi L, Connor CE et al (2012) Toward a unified theory of visual area V4. Neuron 74:12-29. https://doi.org/10.1016/j. neuron.2012.03.011

Rosa MGP, Manger PR (2005) Clarifying homologies in the mammalian cerebral cortex: the case of the third visual area (V3). Clin Exp Pharmacol Physiol 32:327-339. https://doi.org/10.111 1/j.1440-1681.2005.04192.x

Rosenke M, Weiner KS, Barnett MA et al (2017) A cross-validated cytoarchitectonic atlas of the human ventral visual stream. Neuroimage. https://doi.org/10.1016/j.neuroimage 2017.02.040

Roska B, Werblin F (2003) Rapid global shifts in natural scenes block spiking in specific ganglion cell types. Nat Neurosci 6:600-608. https://doi.org/10.1038/nn1061

Rottschy C, Eickhoff SB, Schleicher A et al (2007) Ventral visual cortex in humans: cytoarchitectonic mapping of two extrastriate areas. Hum Brain Mapp 28:1045-1059. https://doi.org/10.1002/ hbm. 20348

Sabbah N, Sanda N, Authié CN et al (2017) Reorganization of early visual cortex functional connectivity following selective peripheral and central visual loss. Sci Rep 7:1-19. https://doi. org/10.1038/srep43223

Safran AB, Achard O, Duret F, Landis T (1999) The "thin man" phenomenon: a sign of cortical plasticity following inferior homonymous paracentral scotomas. Br J Ophthalmol 83:137-142. https ://doi.org/10.1136/bjo.83.2.137

Sahel J-A, Marazova K, Audo I (2015) Clinical characteristics and current therapies for inherited retinal degenerations. Cold Spring Harb Perspect Med 5:a017111. https://doi.org/10.1101/cshpe rspect.a017111

Salimi-Khorshidi G, Douaud G, Beckmann CF et al (2014) Automatic denoising of functional MRI data: combining independent component analysis and hierarchical fusion of classifiers. Neuroimage 90:449-468. https://doi.org/10.1016/j.neuroimage.2013.11.046

Shapiro SS, Wilk MB (1965) An analysis of variance test for normality. Biometrika 52:591-611. https://doi.org/10.1093/biome $\mathrm{t} / 52.3-4.591$

Sokunbi MO, Staff RT, Waiter GD et al (2011) Inter-individual differences in fMRI entropy measurements in old age. IEEE Trans Biomed Eng 58:3206-3214. https://doi.org/10.1109/ TBME.2011.2164793

Sokunbi MO, Fung W, Sawlani V et al (2013) Resting state fMRI entropy probes complexity of brain activity in adults with ADHD. Psychiatry Res Neuroimaging 214:341-348. https://doi. org/10.1016/j.pscychresns.2013.10.001

Stargardt K (1909) Über familiäre, progressive Degeneration in der Maculagegend des Auges. Albr von Graefes Arch für Ophthalmol 71:534-550. https://doi.org/10.1007/BF01961301

Stüber C, Morawski M, Schäfer A et al (2014) Myelin and iron concentration in the human brain: a quantitative study of MRI contrast. Neuroimage 93:95-106. https://doi.org/10.1016/j.neuroimage .2014 .02 .026

Szikra T, Trenholm S, Drinnenberg A et al (2014) Rods in daylight act as relay cells for cone-driven horizontal cell-mediated surround 
inhibition. Nat Neurosci 17:1728-1735. https://doi.org/10.1038/ nn. 3852

Thiebaut de Schotten M, Urbanski M, Batrancourt B et al (2016) Rostro-caudal architecture of the frontal lobes in humans. Cereb Cortex. https://doi.org/10.1093/cercor/bhw215

Timberlake GT, Omoscharka E, Grose SA, Bothwell R (2012) Preferred retinal locus-hand coordination in a maze-tracing task. Investig Opthalmol Vis Sci 53:1810. https://doi.org/10.1167/ iovs.11-9282

Tononi G (1998) Complexity and coherency: integrating information in the brain. Trends Cogn Sci 2:474-484. https://doi.org/10.1016/ S1364-6613(98)01259-5

Tsukamoto Y, Morigiwa K, Ueda M, Sterling P (2001) Microcircuits for night vision in mouse retina. J Neurosci 21:8616-8623

Turano K, Geruschat DR, Stahl JW, Massof RW (1999) Perceived visual ability for independent mobility in persons with retinitis pigmentosa. Invest Ophthalmol Vis Sci 40:865-877

Turano KA, Massof RW, Quigley HA (2002) A self-assessment instrument designed for measuring independent mobility in RP patients: generalizability to glaucoma patients. Invest Ophthalmol Vis Sci 43:2874-2881

Turner R, Oros-Peusquens AM, Romanzetti S et al (2008) Optimised in vivo visualisation of cortical structures in the human brain at 3 T using IR-TSE. Magn Reson Imaging 26:935-942. https://doi. org/10.1016/j.mri.2008.01.043

Tustison NJ, Avants BB (2013) Explicit B-spline regularization in diffeomorphic image registration. Front Neuroinform 7:39. https:// doi.org/10.3389/fninf.2013.00039

Tustison NJ, Cook PA, Klein A et al (2014) Large-scale evaluation of ANTs and FreeSurfer cortical thickness measurements. Neuroimage 99:166-179. https://doi.org/10.1016/j.neuroimage .2014.05.044

Ungerleider LG, Desimone R (1986) Projections to the superior temporal sulcus from the central and peripheral field representations of V1 and V2. J Comp Neurol 248:147-163. https://doi. org/10.1002/cne.902480202

Van Dijk KRA, Sabuncu MR, Buckner RL (2012) The influence of head motion on intrinsic functional connectivity MRI. Neuroimage 59:431-438. https://doi.org/10.1016/j.neuroimage 2011.07.044
Vanduffel W (2002) Extracting 3D from motion: differences in human and monkey intraparietal cortex. Science 298:413-415. https:// doi.org/10.1126/science. 1073574

Volgyi B (2004) Convergence and segregation of the multiple rod pathways in mammalian retina. J Neurosci 24:11182-11192. https:// doi.org/10.1523/JNEUROSCI.3096-04.2004

Wagstyl K, Ronan L, Goodyer IM, Fletcher PC (2015) Cortical thickness gradients in structural hierarchies. Neuroimage 111:241250. https://doi.org/10.1016/j.neuroimage.2015.02.036

Westlye LT, Walhovd KB, Dale AM et al (2010) Differentiating maturational and aging-related changes of the cerebral cortex by use of thickness and signal intensity. Neuroimage 52:172-185. https ://doi.org/10.1016/j.neuroimage.2010.03.056

Wheatstone C (1962) On some remarkable and hitherto unobserved phenomena of binocular vision. Optom Wkly 53:2311-2315

Whiteside TCD (1957) Effect of empty-field myopia upon the minimum visual angle for a distant target. In: The problems of vision in flight at high altitude. Butterworths Scientific, London, pp 105-112

Wittich W, Faubert J, Watanabe DH et al (2011) Spatial judgments in patients with retinitis pigmentosa. Vision Res 51:165-173. https ://doi.org/10.1016/j.visres.2010.11.003

Woo SJ, Park KH, Ahn J et al (2012) Cognitive impairment in agerelated macular degeneration and geographic atrophy. Ophthalmology 119:2094-2101. https://doi.org/10.1016/j.ophth a.2012.04.026

Yao Y, Lu WL, Xu B et al (2013) The increase of the functional entropy of the human brain with age. Sci Rep 3:1-8. https://doi. org/10.1038/srep02853

Yu L, Xie B, Yin X et al (2013) Reduced cortical thickness in primary open-angle glaucoma and its relationship to the retinal nerve fiber layer thickness. PLoS One 8:e73208. https://doi. org/10.1371/journal.pone.0073208

Zeki S (2003) The processing of kinetic contours in the brain. Cereb Cortex 13:189-202. https://doi.org/10.1093/cercor/13.2.189

Zilles K, Amunts K (2010) Centenary of Brodmann's map-conception and fate. Nat Rev Neurosci 11:139-145. https://doi.org/10.1038/ $\operatorname{nrn} 2776$

Zilles K, Amunts K (2015) Anatomical basis for functional specialisation. In: Uludag K, Ugurbil K, Berliner L (eds) fMRI: from nuclear spins to brain functions. Springer, New York, pp 27-66 